Deutschlands ganze Tugend und Schönheit [....] entfaltet sich erst im Krieg.

Thomas Mann, 1914

Und niemand weiß, wer er ist, was er ist, woher er stammt, welcher Truppenteil, ob er ein Gemeiner war oder Offizier, Arbeiter oder Gelehrter oder Künstler. Ein Vermißter, auf den ein halbes Jahrhundert lang womöglich jemand wartet.

Ret Marut (alias B. Traven), 1915

\title{
Kai Artinger
}

Die weißen Flecken hatten ein Gesicht. Illustrierte Verlustlisten und das Berliner Nachweisebüro im Ersten Weltkrieg

Zu Beginn des Ersten Weltkrieges sah man in der Reichshauptstadt Berlin etwas Merkwürdiges. Das preußische Kriegsministerium brachte die Verlustlisten des deutschen Heeres außen am Sockelgeschoß der ehemaligen Kriegsakademie in der Dorotheenstraße an, um die Bevölkerung über Verwundete, Gefangene, Gefallene und Vermißte zu informieren. Diese Verlustlisten, gedruckt im Zeitungsformat, hoben sich hell von der braunen, rußgeschwärzten Fassade ab. Auf einer Fotografie ist der Anblick festgehalten. Menschengruppen stehen vor den amtlichen Anschlägen und studieren die langen Namenkolonnen. Die Namen waren jeweils mit dem Vermerk "verwundet", "gefallen ", "gefangen " oder "vermißt« versehen. Jeder Eintrag kündete vom Schicksal eines Lebens. Jede Liste gab Auskunft über den Stand der Kampfhandlungen und brachte das Kriegsgeschehen auf eine kurze Form. Zusammengenommen machten alle Listen die Dimension des Krieges sichtbar. Die Verlustzahlen erreichten nicht vorstellbare Höhen. Die Soldaten erlebten den Massentod als Resultat einer industrielle Ausmaße annehmenden Kriegsmaschinerie. Diese Erfahrung war neu im 20. Jahrhundert. Wegen ihrer zentralen Bedeutung wurde sie Teil des kollektiven Gedächtnisses.

Nach dem Krieg, im Jahr 1919, hatte Kurt Tucholsky die Praxis der Verlustlisten in einem bitteren politischen Text kommentiert, der den Titel »Die Flecke« trägt:

"In der Dorotheenstraße zu Berlin steht das Gebäude der ehemaligen

Kriegsakademie. Unten, in guter Mannshöhe, läuft eine Granitlage um das Haus herum, Platte an Platte. Diese Platten sehen seltsam aus; sie sind weißlich gefleckt, der braune Granit ist hell an vielen Stellen ... was mag das sein? Ist er weißlich gefleckt? Aber er sollte rötlich gefleckt sein. Hier hingen, während der großen Zeit, die deutschen Verlustlisten. Hier hingen, fast alle Tage gewechselt, die schrecklichen Zettel, die endlosen Listen mit Namen, Namen, Namen ... Ich besitze die Nr. 1 dieser Dokumente: da sind noch sorgfältig die 
Truppenteile angegeben, wenig Tote stehen auf der ersten Liste, sie war sehr kurz, diese Nr. 1. Ich weiß nicht, wie viele dann erschienen sind - aber sie gingen hoch hinauf, bis über die Nummer tausend. Namen an Namen - und jedesmal hieß das, daß ein Menschenleben ausgelöscht war oder 'vermißt‘, für die nächste Zukunft ausgestrichen, oder verstümmelt, leicht oder schwer ${ }^{1}$."

Der Massentod in den Jahren 1914 bis 1918 überstieg alles bis dahin Bekannte und Vorstellbare. Seine Dimension war qualitativ und quantitativ bewußtseinssprengend. Nie zuvor war eine kriegerische Auseinandersetzung so verlustreich. Nie zuvor hatten kriegführende Nationen alles Wissen, alle Ressourcen, alle Kräfte in den Dienst der Vernichtung und Zerstörung gestellt. Das Destruktionsinteresse wurde totalisiert, eine ganze Gesellschaft wurde ihm untergeordnet und dienstbar gemacht mit dem Ergebnis, daß soviel Menschen ausgelöscht wurden wie in keinem Krieg zuvor. Auf annähernd 13 Millionen Tote belaufen sich die Schätzungen. Auf deutscher Seite starben fast 2 Millionen Soldaten an Krankheit oder Verwundung, rund 100000 wurden offiziell als "vermißt " gemeldet, aber als tot angenommen. Es wurde errechnet, daß in diesem weltweiten Konflikt soviel Menschen starben wie in allen Kriegen zwischen 1790 und 1914 zusammengenommen ${ }^{2}$. Napoleons katastrophaler Rußland-Feldzug mit 400000 toten Soldaten, der bis dahin verlustreichste Krieg in der Geschichte überhaupt, wurde weit in den Schatten gestellt von nur einer einzigen der Materialschlachten des Ersten Weltkrieges: der Sommeschlacht, die von Juli bis November 1916 ausgefochten wurde. Eingeleitet von einem sechstägigen Artilleriefeuer, belegten die britischen und deutschen Armeen eine nur sieben Quadratmeilen große Fläche mit 30 Millionen Granaten. Auf keinem Schauplatz dieses Krieges wurden pro Quadratkilometer mehr Soldaten getötet: insgesamt 1200000 . Diesen grausigen Rekordes nicht genug, verloren die britischen Streitkräfte in der ersten Ypernschlacht im Oktober 1914 an einem einzigen Tag 60000 Männer. Das »Massenschlachthaus « - diese brutale Einschätzung des deutschen Autors Wilhelm Lamszus von 1912 über den zukünftigen Krieg - war Wirklichkeit geworden. Dagegen nahm sich der Krim-Krieg (1854 bis 1856), der erste Stellungskrieg der Neuzeit, mit 752000 getöteten Soldaten als "klein« aus. Und auch der amerikanische Bürgerkrieg mit ca. 635000 Toten und der letzte große kriegerische Konflikt Deutschlands im 19. Jahrhundert, der Deutsch-Französische Krieg (1870/71), mit 150000 französischen und rund 45000 deutschen Gefallenen erreichten nicht diese Ausmaße.

Die im Ersten Weltkrieg gemachte Erfahrung mit dem organisierten Massentod löste einen Schock aus, der bis weit in die 20er Jahre nicht verarbeitet wurde. Dieses Erlebnis erschütterte das alte Bild des Soldatentodes in seinen Grundfesten. Die romantisch verklärte Vorstellung vom heroischen, glorreichen Kampf Mann gegen Mann und der versüßte Traum vom zwar tragischen, aber heldenhaften Ableben bei der Verteidigung des Vaterlandes waren schnell vergessen. Angesichts der 1914 hochmodernen und massenhaft zum Einsatz kommenden Distanzwaffen bei Infanterie und Artillerie wurde der Soldatentod in einer Weise herbeigeführt, die sich von demjenigen auf dem traditionellen Schlachtfeld radikal unterschied. Tatsächlich hatte ja »das « Schlachtfeld aufgehört zu existieren. Die ganze Front war

1 Kurt Tucholsky, Die Flecke, in: Ders., Politische Texte, Reinbek bei Hamburg 1982, S. 67.

2 George L. Mosse, Gefallen für das Vaterland. Nationales Heldentum und namenloses Sterben, Stuttgart 1993, S. 9 f. 
zu einem einzigen Kriegsschauplatz geworden. Überall drohte die Gefahr des Todes. Er trat nicht mehr nur beim unmittelbaren Feindkontakt, bei Offensiven und nächtlichen Patrouillengängen im "Niemandsland", dem Todesstreifen zwischen den feindlichen Stellungen, in Erscheinung. Vorne an der Front herrschte permanent die Gefahr einer tödlichen Verwundung. Der Soldat konnte von der Kugel eines Scharfschützen getroffen oder von einem Explosivgeschoß zerrissen werden. Eine Granatexplosion konnte ihn verschütten und lebendig begraben, oder heimtückisches Giftgas verbrannte seine Atemwege, seine Lunge, seine Haut. Die Gefahr drohte auch aus der Luft. Von feindlichen Flugzeugen abgeworfene Eisenpfeile konnten den Soldaten töten. Das Erscheinungsbild des Todes und Sterbens war mannigfaltig. Nichts schien mehr unmöglich zu sein, alles Unvorstellbare denkbar. Da diese und andere fürchterlichen Tötungs- und Verletzungsarten von einem in der Regel anonymen, unsichtbaren Gegner ausgeführt wurden und massenhaft auftraten, büßte der Soldatentod auch den Rest von verbliebener Heroik ein.

$\mathrm{Zu}$ Beginn des Ersten Weltkrieges sah man die Verluste durchaus noch mit anderen Augen. In den ersten Wochen und Monaten waren die Verluste noch überschaubar und die Listen kurz. Und man glaubte den Versprechungen der Militärs und Politiker von einem schnellen Sieg. Da wogen die Zahlen, bei aller Trauer um die Gefallenen, noch nicht so schwer. Es hat den Anschein, daß das Preußische Kriegsministerium die Verlustlisten nicht ohne "Stolz« öffentlich ausgehängte. Denn nach seiner Lesart - und breite Kreise der Gesellschaft teilten diese - waren es »Heldenlisten «. Soldaten hatten sich für den Erhalt, die Ehre, den Ruhm des Vaterlandes geschlagen und geopfert. Jedoch mit dem Stellungskrieg und den Offensiven stieg die Zahl der Verwundeten, Gefangenen, Gefallenen, Vermißten dramatisch an. Die Militärbehörden zogen aus dieser demoralisierenden Entwicklung die notwendige Konsequenz: Sie stellten die Anschläge ein. Die Fläche des Sockelgeschosses der Preußischen Kriegsakademie hätte auch nicht ausgereicht, um all die Namen aufzunehmen. Statt dessen wurden fortan die Deutschen Verlustlisten nur noch als amtliche Beilagen herausgegeben.

Vor 1914 existierten im Kriegsbild die massenhaft anonym verstorbenen Soldaten und Vermißten nicht. Es war unvorstellbar, daß von einem Gefallenen nicht genügend übrig bleiben oder der Körper so verstümmelt und entstellt sein kann, $\mathrm{da} ß$ eine Identifizierung völlig ausgeschlossen ist. Als unerträgliche Übertreibung wäre wohl auch die Vorstellung zurückgewiesen worden, verletzte und gefallene Soldaten könnten nicht vom Schlachtfeld gerettet werden und müßten dort im Dreck ohne seelischen Beistand sterben. Daß Soldaten bei Gefechten einfach "verlorengehen " konnten und daß für nicht wenige Gefallene das Gefechtsfeld zum ortlosen, später unauffindbaren "Grab« werden würde, war ebenfalls bis August 1914 für die Bevölkerung unwirklich. Zwar kannte man das Phänomen der als »vermißt « gemeldeten Soldaten aus alten Kriegen. Aber diese Gruppe war im Verhältnis zu allen anderen Opfern eine zu vernachlässigende Größe gewesen. In einer vorbereiteten Landtagsnotiz zur Regelung der Vermißtennachforschung in Bayern vom Juni 1916 wird die Fehleinschätzung des Krieges thematisiert:

"Die Art der Kriegsführung im jetzigen Krieg und seine lange Dauer haben der Nachforschung nach Vermißten eine Bedeutung gegeben, die man aus früheren

Kriegen nicht kannte und für die deshalb auch nicht vorgesorgt sein konnte." Die erste Fassung dieser Passage lautete: 
"Im Gegensatz zu den früheren Kriegen führte die Art des jetzigen Krieges, insbesondere der Stellungskampf, dazu, daß nach Gefechten der Verbleib einer Anzahl von Offizieren und Mannschaften nicht mit Sicherheit festgestellt werden konnte. Damit ergab sich eine verhältnismäßig hohe Anzahl sogenannter Vermißter. Daß der moderne Krieg eine derartig neue Erscheinung hervorbringen würde, konnte nicht vorausgesehen werden, infolgedessen war es nicht möglich, von vornherein die entsprechenden Maßnahmen für die Vermißtennachforschung zu treffen ${ }^{3}$."

Im Ersten Weltkrieg mußte man erkennen, daß das alte System der Erkennungsmarken, das die Identifizierung von Gefallenen auf den Schlachtfeldern erleichtern sollte, mangelhaft war. Die Militär- und Zivilbehörden sahen sich mit dem wachsenden Problem der Kriegsvermißten konfrontiert, das sie nicht ignorieren konnten. Sie mußten plötzlich Anstrengungen unternehmen, die sie in früheren Kriegen unterlassen hatten. Sie versuchten die Identität anonym verstorbener Soldaten zu ermitteln, um zumindest deren Hinterbliebene von der quälenden Ungewißheit über den Verbleib des geliebten Menschen zu befreien. Und um die Klärung weiterer existentieller Fragen wie Lohnfortzahlung, Kriegsfürsorge, Vermögensverhältnisse und Rentenansprüche zu ermöglichen, die ohne die Todeserklärung des Vermißten nicht durchgeführt werden konnte. Die Dringlichkeit gerade des letzten Punktes machen die zahlreichen Dissertationen deutlich, die zum Thema "Verschollenheit und Todeserklärung « in den Jahren 1916 und 1917 verfaßt wurden ${ }^{4}$.

"Der gegenwärtige Krieg bringt es naturgemäß mit sich, daß der Tod von Kriegsteilnehmern in zahlreichen Fällen sich nicht zweifelsfrei nachweisen läßt. In der ersten Zeit des Bewegungskrieges konnten bei dem ungestümen Vorwärtsdrängen der Heere die Schlachtfelder nicht immer ordnungsgemäß aufgeräumt werden. Während des Stellungskrieges aber bleiben fast bei jedem Gefecht zwischen den beiderseitigen Schützengräben Tote liegen, die nicht beerdigt werden können. Endlich macht auch die alles vernichtende Wirkung der modernen Kampfmittel die Auffindung und Wiedererkennung der Leichen nicht selten unmöglich. So kommt es, daß die Zahl der Vermißten in diesem Kriege recht groß ist. Die Ungewißheit über das Schicksal der ins Feld Gezogenen ist für die Angehörigen nicht nur der Anlaß beständiger Sorge und quälender Beunruhigung, sondern kann auch in vermögensrechtlicher Beziehung nachteilige Folgen haben. Denn alle Vermißten müßten, wenn nicht eine Sonderregelung der Kriegsverschollenheit durch die Bundesratsverordnung (18. April 1916) erfolgt wäre, zunächst mindestens bis zum Friedensschluß in allen Rechts-

Akte Mkr. 13813, Bayerisches Hauptstaatsarchiv Abt. IV, Kriegsarchiv.

4 Walter Schmidt, Die Rechtsverhältnisse der Vermißten nebst der Bundesverordnung über die Todeserklärung Kriegsverschollener vom 18. April 1916, Berlin 1916; Dietrich Borchers, Die Todeserklärung Kriegsverschollener unter besonderer Berücksichtigung der Bundesratsbekanntmachung vom 18. April 1916, Hannover 1917; Kurt Hauptmann, Die Todeserklärung wegen Kriegsverschollenheit unter besonderer Berücksichtigung der Bundesratsverordnung vom 18. April 1916, Greifswald 1916; Hermann Lelewer, Die Todeserklärung Kriegsverschollener nach der Bundesrats-Verordnung vom 18. April 1916, Posen 1916; Adolf Wesche, Die materiellen Voraussetzungen und das Verfahren der Todeserklärung, insbesonderer Kriegsverschollener, Greifswald 1917; Richard Horn, Die Todeserklärung Kriegsverschollener. Eine Studie nach österreichischen und deutschen Zivilrecht, Wien 1917. 
Yaicle.

Atubefaunt Beritorbeue.

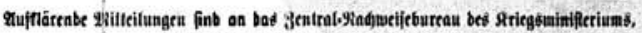

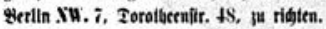
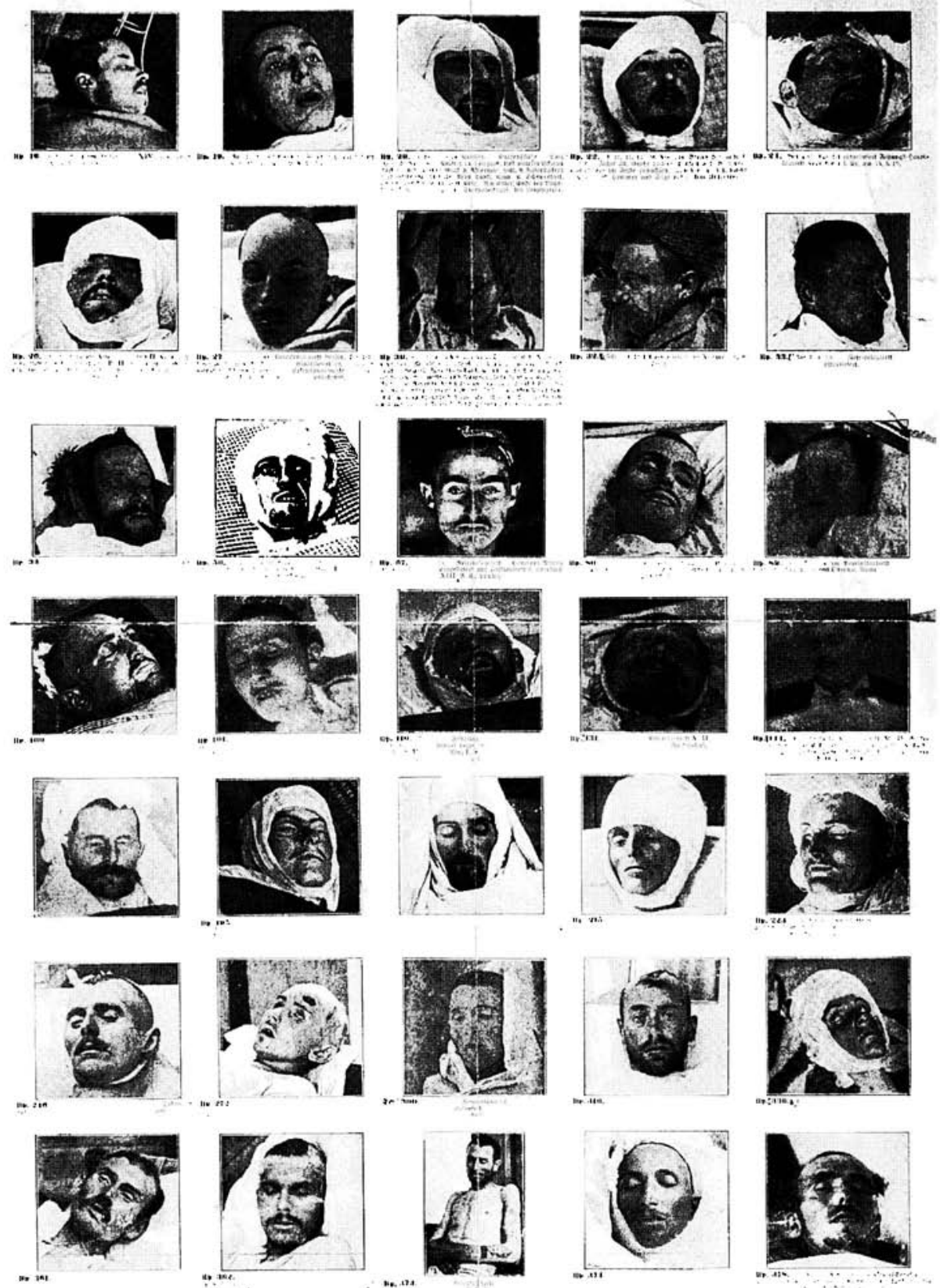

Illustrierte Verlustliste; Quelle: Bibliothek für Zeitgeschichte, Stuttgart 
beziehungen als fortlebend angesehen werden [...] Es liegt auf der Hand, daß es für alle Rechtsverhältnisse von größter Wichtigkeit ist, ob jemand, der im bürgerlichen Leben als Ehemann oder Vater sowie als Gläubiger und Schuldner in Betracht kommt, im Kriege gestorben ist oder als Vermißter rechtlich weiter lebt. Auch für die Erlangung der für den Todesfall zustehenden Gnaden- und Hinterbliebenengebührnisse, der Sterbegelder und Hinterbliebenenrenten nach der Reichsversicherungsordnung sowie für die Verwirklichung von Ansprüchen nach dem Angestelltengesetz ist im allgemeinen die Feststellung des Todes des Kriegsteilnehmers entscheidend. Die neue Bundesratsverordnung wird für weite Kreise insofern von einschneidender Bedeutung werden, als sie die Möglichkeit der Todeserklärung bereits während des Krieges eröffnet ${ }^{5}$.»

Zur Feststellung von Versorgungsansprüchen der Hinterbliebenen war es notwendig, herauszufinden, ob ein Soldat tot ist. Im Falle der Vermißten war das sehr schwierig, da man ermitteln mußte, wo und in welchem Zustand der Soldat sich befand, d.h. ob er verwundet, gefangen oder tot war. Bei den anonym Verstorbenen hatte man zumindest eine Leiche, die eventuell Aufschluß über den Tod eines bisher nur als "vermißt « Gemeldeten bringen konnte. Der Weg, den dann die deutschen Militärbehörden beschritten, um Aufklärung zu erhalten, war drastisch und unter allen am Krieg beteiligten Nationen einzigartig. Man gab den "weißen Flecken « Gesichter. Man zeigte höchst offiziell die Agonie der Kriegsopfer. Illustrierte Verlustlisten wurden gedruckt.

I.

Diese Illustrierten Verlustlisten sind eine ungewöhnliche Bildquelle. Nach dem Ersten Weltkrieg gerieten sie vollständig in Vergessenheit. Erst 1985 entdeckte ein Mitarbeiter der Universitätsbibliothek Tübingen, Peter Berger, eine Kiste mit ungebundenen Deutschen Verlustlisten, die im Zweiten Weltkrieg aus der Berliner Staatsbibliothek ausgelagert und nicht zurückgeführt worden waren. Darin befanden sich auch verschiedene Beilagen zu den Deutschen Verlustlisten. In den 1990er Jahren kam das Material in die Bibliothek für Zeitgeschichte, Stuttgart; dabei ging ein Teil der Illustrierten Verlustlisten verloren. Hans Christian Adam publizierte 1985 einen Aufsatz über das Thema ${ }^{6}$ und veröffentlichte ein Jahr später drei der Tafeln mit den Fotos anonym verstorbener Soldaten ${ }^{7}$. Er fotografierte alle damals im Tübinger Besitz befindlichen Illustrierten Verlustlisten ${ }^{8}$. Adams Beiträge sind bis heute die einzigen zu diesem Thema geblieben. Das mag auch der Grund dafür sein, daß diese Bilder in der Geschichtswissenschaft, selbst bei Spezialisten des Ersten Weltkriegs, fast unbekannt geblieben und demzufolge als Illustrations-

Schmidt, Rechtsverhältnisse (wie Anm. 4), S. 5 f.

Hans Christian Adam, Totensuche. Illustrierte Verlustlisten aus dem Ersten Weltkrieg, in: Fotogeschichte. Beiträge zur Geschichte und Ästhetik der Fotografie, 5 (1985), H. 16, S. $37-44$.

7 Hans Christian Adam, Wer gibt Auskunft?, in: Der Alltag, Berlin 1985, Nr. 1, S. 85 ff.

8 Abzüge aller seiner Negative sind jetzt im Bildarchiv des Deutschen Historischen Museum Berlin zugänglich. 
material in Geschichtsbüchern nicht zu finden sind. Eigene Nachforschungen in der Berliner Staatsbibliothek, Unter den Linden, und die Recherchen von Bibliothekaren in anderen deutschen Archiven und im österreichischen Staatsarchiv Kriegsarchiv haben ergeben, daß diese Beilagen nicht in allen erhaltenen und zu Büchern gebundenen Deutschen Verlustlisten enthalten, d.h. letztere unvollständig sind. So habe ich in Berlin nur einige der illustrierten Tafeln mit Gegenständen und Fotografien unbekannt verstorbener Soldaten, nicht aber die Tafeln mit den Fotos der anonym Verstorbenen gefunden. Das Bayerische Hauptstaatsarchiv - Kriegsarchiv, das eine vollständige Ausgabe der Deutschen Verlustlisten besitzt, weist nur im Band 8 (6. Juni bis 5. August 1915) eine Illustrierte Verlustliste unbekannt Verstorbener auf. Aufgrund dieser spärlichen Quellenlage läßt sich nicht exakt eingrenzen, in welchem Zeitraum und in welcher Anzahl die Illustrierten Verlustlisten herausgegeben wurden. Es ist zu vermuten, daß die Tafeln mit den anonym Verstorbenen nur im ersten Kriegsjahr hergestellt wurden. Da aber von den drei mir bekannten Tafeln nur eine datiert ist (20. Juli 1915), während die anderen beiden kein Datum aufweisen, wegen der Todesdaten aber ein Erscheinungsdatum im Jahr 1915 oder später zwingend machen, bleibt dies hypothetisch. Es müßte eindeutig bewiesen werden, daß später keine solche Tafeln mehr gedruckt wurden. Die Durchsicht der gebundenen Deutschen Verlustlisten hilft nicht bei der Klärung dieser Frage, da offensichtlich die Tafeln mit anonym Verstorbenen nur im Einzelfall eingebunden wurden (einziges mir bekanntes Beispiel ist die Ausgabe der Deutschen Verlustlisten im Bayerischen Kriegsarchiv).

II.

„Unbekannt Verstorbene«. „Wer sind die Gefallenen?« Unter diesen Überschriften der Illustrierten Verlustlisten starrt den Betrachter vieldutzendfach die Agonie der Kriegstoten an. Schmerzverzerrte, offene Münder, geschlossene, gebrochene, starre, leere Augen, in Mullbinden gefaßte Antlitze. Alle Porträtfotografien zeigen die Köpfe von auf dem Transport oder im Lazarett anonym verstorbenen Soldaten. Zwischen 15 und 35 Fotos wurden auf einer Seite zusammengefaßt. Nur wurde hier nicht nach Lebenden, sondern nach der Identität von Toten gefahndet. Die Fotos sind mit Registraturnummern versehen und führen in einer kurzen Legende das Todesdatum, den Ort des Ablebens und weitere sachdienliche Hinweise auf. Diese Sonder-Verlustlisten wurden als separate Beilagen im Zeitungsformat gedruckt und den Deutschen Verlustlisten beigelegt. Der öffentliche Aushang der Tafeln war verboten. Aber sehen sollten die Menschen sie schon. Denn wie sonst hätten.die unbekannten Männer identifiziert werden sollen? Der Nachdruck der Blätter war ausdrücklich gestattet und erwünscht. Für 15 Pfennig einschließlich Porto konnten sie bei der Druckerei bestellt werden.

Aller Wahrscheinlichkeit nach wurden die Verlustlisten vor der Reform des Erkennungsmarkensystems veröffentlicht. In der Regel handelt es sich bei den Dargestellten um verstorbene Soldaten, die in den Kriegsmonaten des Jahres 1914 und 1915 bewußtlos oder tot in ein Lazarett eingeliefert worden waren. Das legt den Schluß nahe, die Militärbehörde habe im ersten Kriegsjahr zu dem Mittel der 
Illustrierten Verlustlisten gegriffen, um anonyme Verstorbene zu identifizieren. Es war genau dieser Zeitraum, in dem noch mit den "alten" Erkennungsmarken gearbeitet wurde.

Die Erkennungsmarke war ein »Blechtäfelchen mit Angabe des Truppenteils und der Nummer der Kriegsstammrolle des Inhabers, [das] jeder deutsche Soldat und (einschließlich Offiziere) im Felde an einer Schnur um den Hals auf bloßem Leibe behufs Feststellung der Persönlichkeit bei Verwundung " (Brockhaus Conversations-Lexikon, 1902) zu tragen hatte. Auf der Erkennungsmarke war zwar die Abkürzung der Formation, es waren aber nicht Name und Wohnort des Trägers verzeichnet. In der Anfangszeit des Ersten Weltkriegs wiesen die verwendeten Marken hinsichtlich ihrer Beschriftung generell nur Truppenteil, Untereinheit (Kompanie, Batterie, Eskadron) und Matrikelnummer des Inhabers auf. Das hatte zur Folge, daß "angesichts der Vielzahl der im Ersten Weltkrieg aufgestellten Formationen (insgesamt standen auf deutscher Seite von 1914 bis 1918 13,25 Millionen Männer unter Waffen, von denen jeder eine Erkennungsmarke besaß) und der hieraus wiederum resultierenden Abkürzungsvielfalt bei den Beschriftungen der Erkennungsmarken « eine vollständige Erfassung sämtlicher jemals verwendeter Abkürzungen wohl nicht erreicht und damit ihre Aufschlüsselung erheblich erschwert oder sogar unmöglich gemacht wurde'.

Als Ausrüstungsgegenstand scheint die Erkennungsmarke ab 1869 allgemein in der preußischen Armee, d.h. in der deutschen Armee ausschließlich der württembergischen, sächsischen und bayerischen Kriegstruppen eingeführt worden zu sein. Davor waren die einfachen Soldaten, wenn sie nicht von Kameraden identifiziert werden konnten, oft namenlos auf dem Schlachtfeld gestorben und in Massengräbern beerdigt worden. Erst Mitte des 19. Jahrhunderts setzte sich der Gedanke durch, mit Hilfe des Systems der Erkennungsmarke die Identifizierung des einzelnen Soldaten zu ermöglichen. Im Ersten Weltkrieg stieß das alte System an seine Grenzen. Es war nicht für das Massenheer ausgelegt und erschwerte die effiziente Registrierung der Toten.

Ein weiteres Problem war ganz profaner Natur. Die deutschen Soldaten trugen mitunter die Erkennungsmarken nicht vorschriftsmäßig, also um den Hals, sondern befestigten sie zum Beispiel am Hosenträger oder Knopfloch des Waffenrocks oder bewahrten sie in der Innentasche des Waffenrocks auf. »Nach Aussage eines Kriegsteilnehmers war dies auch in der Praxis der Fall, da die um den Hals baumelnde Erkennungsmarke oft bei bestimmten Dienstverrichtungen hinderlich war ${ }^{10}$. Die Konsequenz war, daß die Wahrscheinlichkeit, die Marke durch Gewalteinwirkungen zu verlieren, stark anstieg. Aber selbst beim ordnungsgemäßen Tragen konnte die Marke zerstört werden wie etwa in dem Fall eines Verwundeten, dem das um den Hals getragene Blechschildchen durch eine Kugel »in die Lunge und noch weiter hinab gerissen " worden war ${ }^{11}$.

Das Preußische Kriegsministerium suchte die Mängel durch die Einführung einer neuen Erkennungsmarke zu beseitigen. Im Juli 1915 erließ es entsprechende Bestimmungen:

9 Peter Meinlschmidt, Die deutschen Erkennungsmarken des Ersten Weltkrieges. Vor- und Entwicklungsgeschichte des Ersten Weltkrieges im Rahmen der Mobilmachung und des Heeresaufwuchses 1914-1918, Düngenheim (Selbstverlag) 1992, S. 2.

10 Ebd., S. 19.

11 Paul Dorsch, Lazarettbilder aus den ersten Jahren des Weltkrieges, Calw, Stuttgart 1915, S. 18. 
»Um die Feststellung Gefallener mit Sicherheit zu ermöglichen, wird in Abänderung der Heeresordnung [...] folgendes bestimmt:

1. Für Ersatzmannschaften usw., die auf den Kriegsschauplatz nachgesandt werden, wird die Erkennungsmarke von dem Ersatztruppenteil, bei dem der Mann eingestellt wird, und zwar unmittelbar nach seiner Einstellung in ihrem oberen Teil versehen mit:

a) dem Vornamen und Familiennamen,

b) dem letzten Wohnort (bei größeren Orten mit Angabe der Straße und Hausnummer,

c) Zeitangabe der Geburt,

d) der Bezeichnung des Ersatztruppenteils in verständlicher Abkürzung [...] $]^{12}$." Wahrscheinlich wurde die alte Marke bis September 1915 aus den Verkehr gezogen und durch die neue ersetzt. Doch damit war offensichtlich das Problem nicht zu lösen, denn noch im Januar 1917 verfügte das Kriegsministerium: "Zur Verbesserung des Erkennungsdienstes haben die Truppenteile usw. des Feldheeres alle Offiziere, Unteroffiziere und Mannschaften anzuweisen, ihren Namen und letzten Wohnort in möglichst viele ihnen gehörigen Gegenstände einzuschreiben, einzuheften oder bei metallenen Gegenständen, wie Uhren, einzukratzen ${ }^{13} .{ }^{*}$

Wie sehr die Öffentlichkeit diese Angelegenheit beschäftigte, machen ein Artikel der Wochenschrift »Der Krieg « vom Frühjahr 1916 und eine Bekanntmachung der "Deutschen Uhrmacher-Zeitung" vom August 1916 deutlich:

"Sehr traurig ist für die Angehörigen von Feldzugsteilnehmern, wenn sie plötzlich monatelang, ja jahrelang ohne Nachricht aus dem Felde bleiben. Der Gatte, der Sohn oder Bruder ist und bleibt 'vermißt . Der Truppenteil konnte nur melden, daß der Betreffende noch diese oder jene Gefechte mitgekämpft habe, seit dem Tage jedoch nicht mehr aufzufinden gewesen sei. Oft läßt sich aus der Art des Gefechts, ob Rückzugs- oder Patrouillenkämpfe ausgefochten wurden, auf Gefangennahme schließen. Melden jedoch die Vermittlungsstellen nach gründlichen Nachforschungen, daß sich weder in den Gefangenenlagern ein Mann des angegebenen Namens befindet, so muß damit gerechnet werden, daß der Betreffende unerkannt den Heldentod auf dem Schlachtfeld oder in bewußtlosem Zustand in einem Lazarett Aufnahme fand, wo er verschied. Natürlich kann es auch Ausnahmefälle geben, wo ein seit langem Vermißter, ein Totgeglaubter, plötzlich wieder auftaucht, und diese Zeilen sollen durchaus nicht Hoffnungsgedanken zunichte machen. Sie wollen nur zeigen, welches Verdienst sich die deutsche Militärbehörde durch die Ausgabe von Verlustlisten überhaupt und insbesondere durch die neue Vervollkommnung unserer Militärerkennungsmarke erworben hat. Die Militärerkennungsmarke bietet bei der Wirkung unserer modernen Kampfmittel oft die einzige Möglichkeit, die Persönlichkeit eines Bewußtlosen oder Gefallenen festzustellen. Auf die Aussagen von Kameraden, die im letzten Gefecht Seite an Seite mitkämpften, ist nicht mit Sicherheit zu bauen. Verwechslung der Namen - insbesondere bei

12 Kriegsministerium Nr. 1085/7.15.B3, Nr. 594 Änderung der Bestimmungen über Erkennungsmarken, Berlin, 28.7.1915, zit. nach Meinlschmidt, Erkennungsmarken (wie Anm. 9), S. 21.

13 Mkr. 13813: Kriegsministerium, Nr. 261/12, Berlin, 18.1.1917, Bayerisches Hauptstaatsarchiv Abt. IV, Kriegsarchiv. 
Leuten, die noch nicht lange in der Kompanie sind -, die Aufregungen des Gefechts, das beschränkte Sichtfeld, geben zu Täuschungen Anlaß. Dazu kommt oft ein Vermischen der Truppenverbände oder ein Absuchen des Schlachtfeldes durch andere Truppen. Besonders erschwerend war bisher der Persönlichkeitsnachweis von Offizieren und Mannschaften, die im Felde zu anderen Truppenteilen kommandiert waren. All diesen Übelständen hofft man, durch die neue Erkennungsmarke besser entgegentreten zu können ${ }^{14}$."

Die Uhrmacher erklärten ihre Bereitschaft, die Identitätsklärung "gefallener Helden, bei deren Auffindung die Erkennungsmarke fehlte«, über die bei den Gefallenen gefundenen Taschenuhren zu versuchen ${ }^{15}$.

Es ist $\mathrm{zu}$ vermuten, da $\mathrm{B}$ die meisten der fotografierten Toten der Illustrierten Verlustlisten entweder ohne ihre oder mit einer nicht entschlüsselbaren Erkennungsmarke zum Sanitätstransport und ins Lazarett gelangten. Eine Auskunft über ihre Identität konnten sie wegen ihres Zustandes - Bewußtlosigkeit, Tod - nicht geben. Zwangsläufig wurden diese Unbekannten weiterhin in den Verlustlisten als "vermißt« geführt, ohne daß $\operatorname{man}$ es korrigieren konnte. Die Illustrierten Verlustlisten sollten Abhilfe schaffen.

Ein ärztliches Rapport- und Berichtswesen sorgte dafür, daß die Verluste gemeldet wurden. In der Regel berichteten die Truppen und Formationen des Feldund Besatzungsheeres über die eigenen Angehörigen nach dem Eintritt eines Verlustes. Das geschah nach jedem Gefecht. Sie sandten Namenslisten der Gefallenen, Verwundeten, außerhalb der Lazarettbehandlung Gestorbenen, Vermißten und Gefangenen, und an Ortsbehörden oder Heilanstalten des Feindes zur ärztlichen Behandlung abgegebenen Kranken an das Zentralnachweisebüro des Preußischen Kriegsministeriums.

"Sind bei einem Truppenteil im Felde Verluste eingetreten und steht der Tod des einzelnen Kriegsteilnehmers fest, so werden durch den Regimentskommandeur Sterbefallanzeigen an die Ersatztruppenteile erstattet. Auf Grund dieser Anzeigen erfolgt dann die standesamtliche Beurkundung des Todes bei demjenigen Standesamt, in dessen Bezirk der Verstorbene seinen letzten Wohnsitz gehabt hat. Aus dieser im Sterberegister enthaltenen Eintragung erhalten Angehörige des Verstorbenen kostenfrei einen beglaubigten Auszug, auf Grund dessen sie dann die zur Erlangung über Gebührnisse weiter erforderlichen Schritte tun können. In vielen Fällen aber ist der Truppenteil, dem der Kriegsteilnehmer zuletzt angehört hat, nicht in der Lage, den Tod dienstlich zu beglaubigen, sondern muß ihn als ,vermißt , führen. Gelingt es dem Truppenteil auch nach Verlauf weiterer Zeit nicht, Bestimmtes über den Verbleib des Vermißten festzustellen, so pflegt er sich mit dem zuständigen Zentral-Nachweisebureau in Verbindung zu setzen, das die Verlustlisten über gefallene, verwundete, vermißte und gestorbene Kriegsteilnehmer bearbeitet ${ }^{16}$. . Das Zentralnachweisebüro und die Zentralstelle für Nachlaßsachen sammelten sämtliche Informationen und erstellten daraus die Deutschen Verlustlisten. Über diese Militärbehörde ist leider nicht sehr viel bekannt. Im »Sanitätsbericht über

14 Hans Schipper, Militärerkennungsmarken, in: Der Krieg 1914/17, Nr. 120, S. 952; zit. nach Meinlschmidt, Erkennungsmarken (wie Anm. 9), S. 28 f.

15 Deutsche Uhrmacher-Zeitung, 40 (1916), Nr. 16, S. a.

16 Schmidt, Rechtsverhältnisse (wie Anm. 4), S. 7. 
das Deutsche Heer" aus dem Jahre 1935 findet sich immerhin eine kurze Beschreibung über diese Institution ${ }^{17}$.

Danach gründete sich die Einrichtung des Nachweisebüros der Kriegerverluste in der Hauptsache auf die Vorschriften der Kriegssanitätsordnung, der Heerordnung, der Marineordnung, der Genfer Konvention über die Gebräuche des Landkrieges und der Haager Landkriegsordnung. Schon diese Dienstvorschriften und Anordnungen legten fest, daß das Nachweisebüro zwei voneinander getrennte Aufgaben verrichten sollte: Erstens den Nachweis der Verluste des eigenen Heeres, und zweitens den Nachweis über Verbleib und Schicksal kriegsgefangener Feinde.

Fünf Nachweisebüros wurden 1914 eingerichtet: das Zentralnachweisebüro in Berlin, das Zentralnachweisebüro der Marine, ebenfalls in Berlin, und die Nachweisebüros in München, Dresden und Stuttgart. Die letzten drei waren Abteilungen der betreffenden Kriegsministerien bzw. des Reichsmarineamtes. Im Berliner Zentralnachweisebüro arbeiteten Vertreter der Landesnachweisebüros, die für den Nachrichtenaustausch sorgten.

Die einzelnen Nachweisebüros waren fast identisch aufgebaut. Sie zerfielen in vier Referate:

I: Bearbeitung der fünftägigen Meldungen der Lazarette. Auskunftserteilung.

II: Sammlung und Veröffentlichung der Verlustlisten. Auskunftserteilung.

III: Sammlung der Nachrichten über Angehörige der feindlichen Heere bzw. Kriegsgefangene. Auskunftserteilung.

IV: Vermittlung und Beurkundung der Sterbefälle. Öffentliche Auskunftstelle für mündliche Auskunftserteilung.

Im Berliner Zentralnachweisebüro waren zu Beginn 193 Mitarbeiter beschäftigt. Doch die Behörde war viel zu klein konzipiert. Sie drohte mit der Fortdauer des Krieges im Listenwesen zu ersticken. Der Aufbau großer Datenbanken in Form von Karteien wurde notwendig, um den Inhalt der Listenmeldungen schneller abrufen zu können. Der Umfang der schriftlichen Arbeiten der Behörde wuchs auf ein ungeheuerliches Maß an. Die Nachforschungen nach Vermißten in Verbindung mit dem Roten Kreuz, die Mitwirkung bei dem gerichtlichen Todeserklärungsverfahren vor den Amtsgerichten, die Erteilung von militärdienstlichen Bescheinigungen aller Art, die Vermittlung der standesamtlichen Beurkundung von Sterbefällen und viele andere Aufgaben wurden uferlos. Ende 1918 hatte das Zentralnachweisebüro 2800 Beschäftigte. Allein diese Zahl veranschaulicht den gewaltigen verwaltungstechnischen Aufwand bei der Bearbeitung der Verluste.

Das Preußische Kriegsministerium scheint die einzige deutsche Militärbehörde gewesen zu sein, die zu Beginn des Krieges Verlustlisten aushängte. Von anderen Ländern ist dies nicht bekannt. Üblich war, die Listen in amtlichen Organen oder in Zeitungen abzudrucken. In Bremen konnte man die Listen in den Zeitungen sehen. Die Württembergischen Verlustlisten erschienen im "Staatsanzeiger", allerdings nur bis 1915. Dann stellte man den Abdruck ein. Über die genauen Gründe ist nichts bekannt.

17 Sanitätsbericht über das Deutsche Heer, Bd 3: Die Krankenbewegung bei dem Deutschen Feld- und Besatzungsheer im Weltkriege 1914/1918, Berlin 1934, S. $161 \mathrm{f}$. 
III.

Aus dem Blickwinkel des heutigen Betrachters erscheinen die Illustrierten Verlustlisten als "Schreckensbilder ". Völlig frei von dieser negativen Aura waren sie auch zu ihrer Zeit nicht, wie das Aushängungsverbot beweist. Am treffendsten charakterisiert man die Gefühle beim Anblick der Porträts der anonym verstorbenen Soldaten mit "zwiespältig «. Obwohl alle Fotos keine verstümmelten und entstellten Körper zeigen - was der Zielsetzung auch entgegengestanden hätte -, ist die eingefangene Agonie schwer zu übersehen.

Bis heute ist in der einschlägigen Literatur immer wieder zu lesen, die offiziellen deutschen Kriegsfotografen und Soldaten hätten nie die eigenen, sondern nur die Gefallenen der Gegenseite fotografiert ${ }^{18}$. Bodo von Dewitz wies darauf hin, daß insbesondere die Amateurfotografen unter den Soldaten die Realität des Todes nicht aussparten, solange es die Toten des Feindes waren. Dagegen sei der Tod der eigenen Soldaten indirekt in Bildern von Heldengräbern, Beerdigungen oder ganzen Friedhofsanlagen festgehalten worden.

"Die soldatische Amateurfotografie hat mit solchen Grabesfotografien nicht nur die Vorkommnisse des eigenen Sterbens tabuisiert, sie hat in besonders anschaulicher Weise dazu beigetragen, den Heldentod zu zelebrieren, indem sie dessen > Realität ‘ in Fotografien von Heldengräbern festgehalten hat. Solche Fotografien konnten dann auch Reliquienfunktionen erfüllen [...] Fotografien der Leichen feindlicher Soldaten muß es in großer Zahl gegeben haben. Denn in den Zeitschriften der Fachpresse fühlten sich viele Verfasser bemüßigt, zu solchen Motiven der `Schrecken des Krieges< Stellung zu nehmen [...]: >Als ausgesprochener Gegner möchte ich mich zu einer Art von Bildern bekennen, welche manche Lichtbildner unter dem Motto 'Schrecken des Krieges' aufnehmen; das sind Photographien gefallener feindlicher oder gar eigener Soldaten, ja von ganzen Leichenfeldern oder mit Leichen gefüllter Gräben [...] Ob derartige Aufnahmen sehr geschmackvoll sind, möchte ich der allgemeinen Beurteilung anheimstellen, ich finde sie keineswegs am Platze ${ }^{19}$."

Ganz allgemein lehnten die Vertreter der künstlerischen Amateurfotografie, die nicht als Soldaten eingezogen worden waren, die Bilder von Toten ab und hielten Schmerz und Tod für kein geeignetes Objekt. Die Fotoamateure an der Front sahen das grundsätzlich anders und teilten diese moralische Ablehnung nicht. Jedoch nahmen sie das Bild der gefallenen Kameraden nur dann auf, wenn es einen "geordneten Tod « wiedergab und der Soldatentod als »Heldentod « interpretiert werden konnte.

Die Illustrierten Verlustlisten sind das ungewöhnliche Beispiel dafür, daß es zumindest einige Ausnahmen gab und das deutsche Heer durchaus seine eigenen Toten fotografierte. Es waren nicht die Gefallenen an der Front, sondern die auf den Sanitätstransporten und im Lazarett ihren schweren Verwundungen erlegenen Soldaten. im Ersten Weltkrieg, München 1989, S. 251-262. 
Wie aber ist es möglich, daß eine Behörde ganz offiziell Fotos verbreitete, die der Kategorie "Schreckensbilder " zugeordnet werden können, ohne dabei in Gefahr zu geraten, die eigene Haltung zu untergraben?

Eine Erklärung könnte sein, daß es eine Frage des Wahrnehmungskontextes ist, in dem die Bilder stehen. So paradox es klingt, die Illustrierten Verlustlisten entsprachen in den ersten beiden Kriegsjahren dem vorherrschenden Kriegsbild. Aus ihnen konnte durchaus die Botschaft des folgenden Zeitungsartikels des Stuttgarter »Deutschen Volksblattes« vom 18. Oktober 1915 gelesen werden:

"Durchhalten!

Über ein Jahr stehen wir im Riesenkampf um unser Dasein. Furchtbar sind die Opfer, die unser Volk hat bringen müssen; sie sind ihm erträglich geworden in dem stärkenden Bewußtsein, daß sie nicht umsonst gebracht wurden, und daß die Zukunft die Leiden und Beschwernisse der harten Gegenwart entgelten wird. Wir wissen unserem Heere tiefen, unauslöschlichen Dank für seine herrlichen Taten, die uns die Schrecken des Krieges im eigenen Land erspart haben; jedem einzelnen braven Soldaten danken wir für seinen Opfer- und Heldenmut ${ }^{20}$."

Blickt man auf die Zensurbestimmungen, erkennt man, daß fotografische und künstlerische »Schreckensbilder « nicht grundsätzlich verboten waren. Nach den Bestimmungen des Zensurbuches für die deutsche Presse von 1917, das kurze Anweisungen und Richtlinien vom Preußischen Kriegsministerium, dem stellvertretenden Generalstab der Armee und der Oberzensurstelle des Kriegspresseamtes enthielt, unterlagen Kunstbilder dann einer Vorzensur, wenn sie Darstellungen militärischer Art zeigten. Was solche im einzelnen waren, wurde nicht ausgeführt. Geradezu »freizügig « klingt der nachstehende Passus: "Künstlerische Darstellungen: Skizzen, Zeichnungen, Malereien, plastische Darstellungen usw. zu Ausstellungs- oder Einzelverkaufszwecken sind in weitestem Umfange zur Veröffentlichung zugelassen, selbst wenn sie den ganzen Ernst des Krieges (Kampfszenen, Tote, Schwerverwundete) zeigen. Wünscht die Zensurbehörde eine Massenverbreitung auszuschließen, so versieht sie den Freigabevermerk mit einen entsprechenden Zusatz.«

Selbst wenn man bedenkt, daß sich diese Bestimmung erst 1917 im Zensurbuch finden läßt - wobei das Handbuch den Versuch staatlichen Entgegenkommens gegenüber der Presse darstellte, um deren Arbeit unter den Bedingungen des Belagerungszustandes und der militärischen Aufsicht zu "erleichtern « -, gibt sie einen interessanten Einblick in den Umgang der Zensurbehörde mit Schreckensbildern. Obwohl das Massensterben bereits in sein drittes Jahr gegangen war, unterlagen Bilder von Toten und Schwerverwundeten keinem generellen Tabu, es sei denn, die Freigabe wurde von der Militärbehörde aus bestimmten Gründen verweigert. An anderer Stelle wurden diese Gründe genannt, aber auch sie lassen eine gewisse Freizügigkeit erkennen. Die relevanten Sonderbestimmungen der Bildzensur lauteten:

"1. Künstlerische Darstellungen (Skizzen, Zeichnungen, Malereien) zu Ausstellungen- bzw. Einzelverkaufszwecken sind zur Veröffentlichung zugelassen, selbst wenn sie den ganzen Ernst des Krieges zeigen. Übertriebene Darstellungen der Schrecken des Krieges oder solche, die von unseren Feinden 
zu unserer Kriegsführung nachteiliger Auslegung benutzt werden können, sind jedoch zu verhindern.

Um eine Massenverbreitung solcher Darstellungen zu verhüten, die zwar zulässig, aber bei Einzelverkauf oder Massenverbreitung geeignet sind, auf die Volksstimmung ungünstig einzuwirken, ist der Freigabevermerk mit einem entsprechenden Zusatz zu versehen, bei Sammelwerken: 'Einzelwiedergabe verboten<, bei Einzeldarstellungen: >Postkarten- oder anderweitige Vervielfältigung nicht gestattet. [ [...]

4. Abbildungen toter oder verwundeter Soldaten sind von der Veröffentlichung auszuschließen, wenn die Züge der Dargestellten so deutlich erkennbar sind, daß ein Wiedererkennen möglich ist.

5. Abbildung verwundeter, verstümmelter, erblindeter deutscher Soldaten sind zugelassen, wenn sie den Zweck erkennen lassen, weniger das Leiden solcher Kriegsbeschädigter als ihre Pflege und ihr Wohlergehen zur Darstellung zu bringen ${ }^{21}$."

Die in der 1. Sonderbestimmung verwendeten Begriffe sind zwar unpräzise und lassen einen Interpretationsspielraum zu - wann zum Beispiel wurden Darstellungen, die »den ganzen Ernst des Krieges« zeigten, zu »übertriebenen Darstellungen des Schreckens «? -, doch bemerkenswerter ist, daß die Wiedergabe der Realität grundsätzlich erlaubt war. Die Sonderbestimmungen 4. und 5. konnten eigentlich nur bei der Fotografie richtig greifen, denn die Kriegsmaler und -zeichner strebten bei der Darstellung von Toten oder Verwundeten nur sehr selten Porträtähnlichkeit an. Die darstellende Kunst des Ersten Weltkriegs belegt das.

Geht man von der Chronologie des Krieges aus, erstaunt die Vorgehensweise der Bildzensur, da man annehmen müßte, daß gerade mit der Dauer des Krieges und der unablässig steigenden Zahl der Opfer Schreckensdarstellungen überhaupt völlig verboten wurden (durchschnittlich fielen auf deutscher Seite täglich 1000 Männer, d.h. in jeder Minute des Krieges wurden drei Soldaten getötet ${ }^{22}$ ). Einer positiven Propaganda im Inland standen solche Bilder ja eigentlich entgegen. Trotzdem gab es in den ersten beiden Kriegsjahren Beispiele, die den Horror darstellten. Man findet sie in der Fotografie, bei den offiziellen Kriegsmalern und bei den Künstlern, die an der Front als Soldaten kämpften. Allerdings stellten auch diese die Toten der Gegenseite, jedoch nicht die eigenen dar. »[...] zeige ich dir ein toder Russe, der schon ganz schwarz angelaufen ist «, schrieb der Soldat Richard Reu im Mai 1915 seiner Frau in einem Feldpostbrief, der auf der Rückseite passend mit dem aquarellierten Bild eines toten Russen versehen ist ${ }^{23}$.

Die Illustrierten Verlustlisten zeigen viele Soldaten, die an Kopfschüssen gestorben waren. Der Schädelschuß bildet im Kriege ein häufiges Vorkommnis, schrieb der Arzt Hermann Simon in seiner kriegschirugischen Skizze »Der Schädelschuß«(Berlin 1916). Das gehäufte Auftreten dieser Verwundung war eine Folge der besonderen Bedingungen des Stellungskrieges. Es verwundert daher

21 Der Erste Weltkrieg. Zeitgenössische Gemälde und Graphik. Veröffentlichung des Bayerischen Armeemuseums, Bd 1, Ingolstadt 1980, S. 19. Hervorhebungen durch Verfasser.

22 Zahlen nach Kurt Kszyk, Entwicklung der Kommunikationskontrolle zwischen 1914 und 1918, in: Heinz-Dietrich Fischer, Pressekonzentration und Zensurpraxis im Ersten Weltkrieg, Berlin 1973, S. 177.

23 Richard Reu, Feldpostbrief, Alter Zeughausbestand, Graphische Sammlung des Deutschen Historischen Museums, Inventarnr. KG 55/17-85. 
nicht, daß Kopfverwundete auch in der bildenden Kunst häufig dargestellt wurden. Doch die Bildaussage fiel sehr unterschiedlich aus. Bei den Malern Albin EggerLienz und Fritz Erler standen die Verbände als Zeichen für Durchhaltevermögen und einen Kampf bis in den Tod, also für »moralische Stärke". In dem berühmten Monumentalgemälde "Krieg" (1915/16) von Egger-Lienz zieht sogar ein Soldat mit Kopfverband ins Gefecht! Das Aquarell "Wo kommst Du her in Deinem roten Kleid? « (1914) von Fritz Erler zeigt den verwundeten Soldaten - auch er hat den Kopf verbunden - von einer heroischen Seite und im Willen ungebrochen. Dagegen betonten Max Beckmann in seiner Zeichnung seines Schwagers Martin Tube und Erich Heckel in einem Holzschnitt zweier Soldaten im Lazarett den Schmerz und die seelische Ernüchterung.

IV.

Es ist deutlich geworden, daß der Soldatentod eine vielschichtige Bedeutung hatte. In den ersten Kriegsjahren war er als Opfer fürs Vaterland und als »Heldentod« positiv besetzt. Vor diesem Hintergrund wird es verständlich, warum die Militärbehörde zuerst die Verlustlisten nicht als bedrohlich für die öffentliche Ruhe und Ordnung ansah. Der Großteil der Bevölkerung erblickte in ihnen nicht primär einen Ausdruck der Kriegsschrecken. Sie galten als Beweis der heroischen nationalen Verteidigungsanstrengungen.

Die Begriffe »Tod « und »Opfer « sind eng miteinander verzahnt. Der Tod des einzelnen erhielt seinen Sinn durch das Opfer für die Allgemeinheit; das Opfer des einzelnen wurde durch die kollektive Anstrengung zu einem gemeinschaftlichen Opfer transzendiert. Zumindest am Anfang machten die Verlustlisten mit den Hunderten von Namen sichtbar, wie groß die gesellschaftliche Anstrengung war. Fast aus jeder Familie aus allen Schichten war ein Sohn oder Vater in den Krieg gezogen.

Erscheinen dem heutigen Betrachter die Illustrierten Verlustlisten als Zeugnisse der Agonie, so wurden sie mit einiger Sicherheit von den Nachweisebüros nicht so gesehen. Denn alle Schrecken, die die Porträtfotos erahnen lassen, wurden überlagert und zurückgedrängt von der Idee des sinnvollen Todes. Es ist anzunehmen, daß die Porttäts der toten Soldaten zwar auch schon damals nicht den Eindruck glorreicher Helden vermittelten, dennoch konnten sie im Rahmen des vorherrschenden Kriegsbildes als Opferbilder und nicht als Zeugnisse sinnlosen Sterbens wahrgenommen werden. So ließe sich erklären, daß diese von der Militärbehörde herausgegebenen »Bilder der Agonie« gerade nicht in erster Linie als Schreckensbilder angesehen wurden. Der individuelle Schrecken des Todes, wie ihn der einzelne erlebt, wurde durch den kollektiven Charakter des massenhaften Opfèrs verdrängt. Der Kontext des Kriegsbildes "neutralisierte « die Tatsache, daß die Fotos Dokumente des dem Sterben vorausgegangenen großen Leidens und der Todesangst sind.

Das Paradoxon der Illustrierten Verlustlisten liegt darin, daß das Kriegsministerium damit eine Art »Normalität « der Situation vortäuschte. Trotz der vielen Toten schien es ganz Herr der Lage zu sein. Man gab sich den Anschein, daß nichts 
unversucht gelassen wird, um die Hinterbliebenen jedes gefallenen Soldaten über dessen Schicksal zu informieren.

Möglicherweise gerieten die Militärbehörden unter der neuartigen Situation in einen Erklärungsnotstand: Sie fühlten sich und waren wohl auch dazu verpflichtet, über die ihnen anvertrauten Männer Rechenschaft zu geben, konnten es in vielen Fällen jedoch nicht. Um das Vertrauen der Bevölkerung in die Glaubwürdigkeit und die Autorität der Militärbehörden nicht zu erschüttern, war es unmöglich zuzugeben, daß der Stellungskrieg Bedingungen erzeugte, die es zuließen, daß ein Soldat einfach "verschwinden ", daß er sogar infolge schwerster Kopfverletzungen bis zur Unkenntlichkeit verstümmelt werden konnte, so daß seine Identität nicht mehr feststellbar war. Dies zuzugeben hätte bedeutet, den veränderten Krieg erklären, den Sinnverlust eingestehen zu müssen. Man hätte offenbaren müssen, daß die Bilder von Helden und vom heldenhaften Kampf anachronistisch geworden waren. Im Heldenkampf gibt es keine anonymen Toten, die Helden haben immer Namen. Die Illustrierten Verlustlisten waren der drastische Versuch, die Würde des Helden und damit den Opferkult, der den Soldatentod legitimierte, aufrechtzuerhalten. Zur Erreichung dieses Zwecks nahm man den Nebeneffekt in Kauf, punktuell die Schrecklichkeit des Todes sichtbar werden zu lassen.

Somit lassen sich die Illustrierten Verlustlisten als Versuch der Militärbehörden interpretieren, in die Unordnung unaufgeklärter Verluste wieder Ordnung zu bringen. Es soll hier keineswegs dem Mythos "preußischen Ordnungssinnes « das Wort geredet werden. Die Einschätzung Hans Christian Adams, die Illustrierten Verlustlisten seien meine Merkwürdigkeit preußischen Ordnungssinnes « ${ }^{24}$, weil kein anderer Kontrahent ein vergleichbares Unternehmen betrieben hat, greift als Erklärung sicherlich zu kurz. Trotzdem ist seine implizite Frage berechtigt, warum nur die Deutschen einen so drastischen Weg einschlugen, um die Identität von anonymen Toten zu ermitteln. Blickt man auf das Feld der bildenden Kunst, könnte eine Erklärung darin liegen, daß es in Deutschland realistischere Darstellungen des Soldatentodes gab als in den anderen kriegführenden Staaten, weil hier gewisse philosophische und künstlerische Voraussetzungen vorlagen.

Abschließend ist festzuhalten, daß das Problem der vermißten und anonymen toten Soldaten für die Militärbehörden auf die Dauer nicht zu bewältigen war. Das Gesetz der großen Zahl, oder anders formuliert: das Massenheer, schuf einfach Fakten. Unter den Verhältnissen der Abnutzungsschlachten ab 1916 war es unmöglich, die Identität aller unbekannten Toten zu ermitteln. In der Schlacht bei Verdun verloren die Deutschen 281000 Männer. Es ist nicht bekannt, wieviele der auf dem Gefechtsfeld verletzten Soldaten schließlich anonym im Lazarett starben. Aber es ist zu vermuten, daß die Erstellung Illustrierter Verlustlisten, hätte man sie in der zweiten Kriegshälfte fortgesetzt, einen Arbeitsaufwand bedeutet hätte, den das Zentralnachweisebüro in Berlin nicht zu leisten vermochte. In früheren Kriegen - die letzte große Schlacht der Deutschen war bei Sedan (1871) - hatte es auch Vermißte und anonym Verstorbene gegeben, doch ihre Zahl war, gemessen an den Dimensionen im Ersten Weltkrieg, klein. In Sedan verzeichneten die Deutschen rund 9000 Tote.

Eine mögliche Schlußfolgerung aus diesen Überlegungen ist, daß die Erscheinung der Illustrierten Verlustlisten im Entwicklungsprozeß vom alten zum modernen, industrialisierten Krieg begründet war. 